\title{
Impact of hydrogen fuelling on confinement properties in radiative improved mode
}

\author{
D Kalupin ${ }^{1}$, P Dumortier ${ }^{2}$, A Messiaen ${ }^{2}$, M Z Tokar ${ }^{1}$, B Unterberg ${ }^{1}$, \\ Geert Verdoolaege $^{3}$, G Van Wassenhove ${ }^{2}$ and R Weynants ${ }^{2}$ \\ ${ }^{1}$ Institut für Plasmaphysik, Forschungszentrum Jülich GmbH, EURATOM Association, D-52425 \\ Jülich, Germany \\ ${ }^{2}$ Laboratoire de Physique des Plasmas/Laboratorium voor Plasmafysica, EURATOM \\ Association, Ecole Royale Militaire, B-1000 Brussels, Belgium \\ ${ }^{3}$ Department of Applied Physics, Ghent University, Rozier 44, 9000 Ghent, Belgium \\ E-mail: d.kalupin@fz-juelich.de
}

Received 2 April 2003, in final form 13 June 2003

Published 18 July 2003

Online at stacks.iop.org/PPCF/45/1501

\begin{abstract}
The radiative improved (RI) mode at TEXTOR is a high confinement regime, which is obtained by the seeding of neon into deuterium plasmas. Recent experiments were aimed to study the influence of external gas fuelling on the confinement properties of the RI mode. In particular, it was found that a hydrogen puff into such plasmas leads to lower confinement compared with the discharges fuelled with the same amount of deuterium gas. This paper attempts to explain the reduction of confinement in RI plasmas with an external hydrogen puff and its relation to the level of impurity concentration, which is a critical parameter for RI mode confinement.
\end{abstract}

\section{Introduction}

The degradation of energy confinement with reduced atomic mass of the working gas is a common feature observed in diverse operational modes in tokamaks. In low $(\mathrm{L})$ and high $(\mathrm{H})$ confinement modes, the energy confinement timescales approximately as a square root of the atomic mass $A_{i}$. In the radiative improved (RI) mode at TEXTOR, the confinement degradation caused by puffing of hydrogen is even stronger than $A_{i}^{0.5}$ [1]. This fact suggests that in the case of the RI mode some other effects, leading to stronger degradation, should be of importance.

A correlation of the confinement quality with the plasma edge properties was found in many tokamak devices. In the RI mode, the degradation of confinement caused by strong deuterium fuelling by a gas puff occurs due to intensification of anomalous transport at the plasma edge accompanied by an increase in the recycling flux [2,3,5]. Experiments done with hydrogen fuelling show a similar correlation of energy confinement with recycling 
flux: higher recycling corresponds to lower confinement [1]. Thus, it is reasonable to suppose that the mechanism leading to confinement degradation is similar in both cases.

This paper discusses the mechanism of confinement deterioration in deuterium plasmas fuelled by hydrogen puffing on the basis of experimental observations on TEXTOR and based on the results of computations done with one-dimensional transport code RITM [4]. By comparing with the case of confinement degradation caused by a strong deuterium puff [3] common limitations for plasma fuelling with a gas puff are considered.

\section{Confinement properties of RI plasmas fuelled by deuterium and hydrogen gas puffs}

As was demonstrated in [1], a hydrogen puff in the RI mode leads to lower electron density and reduced energy confinement compared to discharges fuelled with the same amount of deuterium gas. Figure 1 presents the evolution of energy confinement time in two discharges fuelled during the RI mode stage by hydrogen and deuterium gas puffs. $\tau_{\mathrm{E}}$ is shown as a function of Greenwald number, $n / n_{\mathrm{Gr}}$, increasing during the fuelling, where $n_{\mathrm{Gr}}=I_{\mathrm{p}} / \pi a^{2}$ $\left(10^{20} \mathrm{~m}^{-3}\right), I_{\mathrm{p}}$ is the plasma current in mega-Amperes and $a$ the minor radius in metres. With a hydrogen puff the energy confinement time saturates at a low level and a lower density can thus be finally achieved. It is reasonable to ask: can these results be explained only by the dependence of confinement properties on isotope composition like that observed in $\mathrm{L}$ and $\mathrm{H}$ modes?

As was shown in [7], the main transport channel in the RI mode is due to the dissipative trapped electron (DTE) instability. Therefore, the transport should not, necessarily, have the same dependence on the atomic mass as under $\mathrm{L}$ or $\mathrm{H}$ mode conditions, where the transport is mainly due to ion temperature gradient (ITG) instability [10]. Moreover, $\mathrm{D}_{2} / \mathrm{H}_{2}$ puff experiments were performed in deuterium plasmas with deuterium neutral beam injection, the amount of puffed gas does not exceed a small percentage of total recycling flux and should not change the isotope composition in the centre of plasma significantly, which might have been the reason for confinement deterioration. But the change of the atomic mass can be pronounced at the plasma edge, and thus the transport properties can differ significantly in plasmas with $\mathrm{D}_{2}$ and $\mathrm{H}_{2}$ puffs.

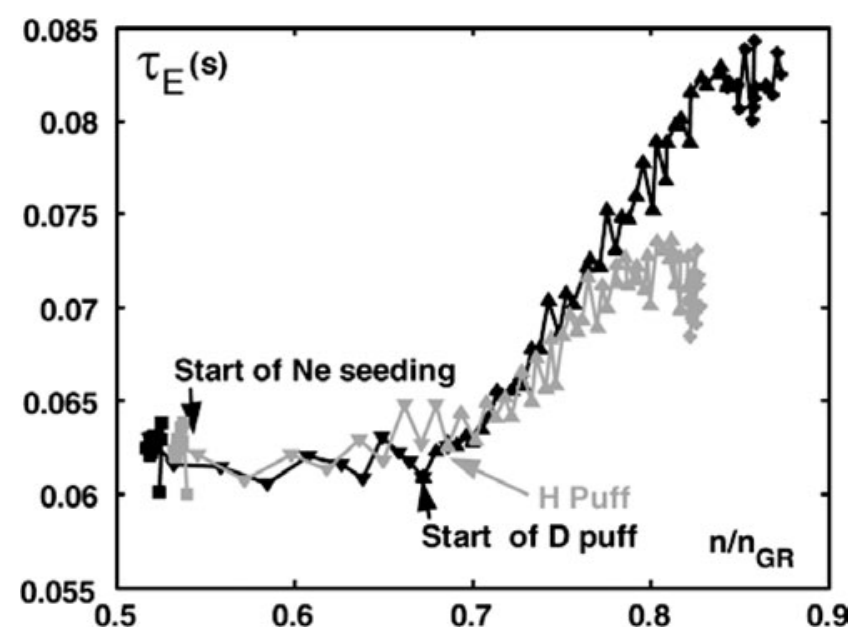

Figure 1. Evolution of the energy confinement time as a function of the Greenwald number with hydrogen and deuterium puffing. 


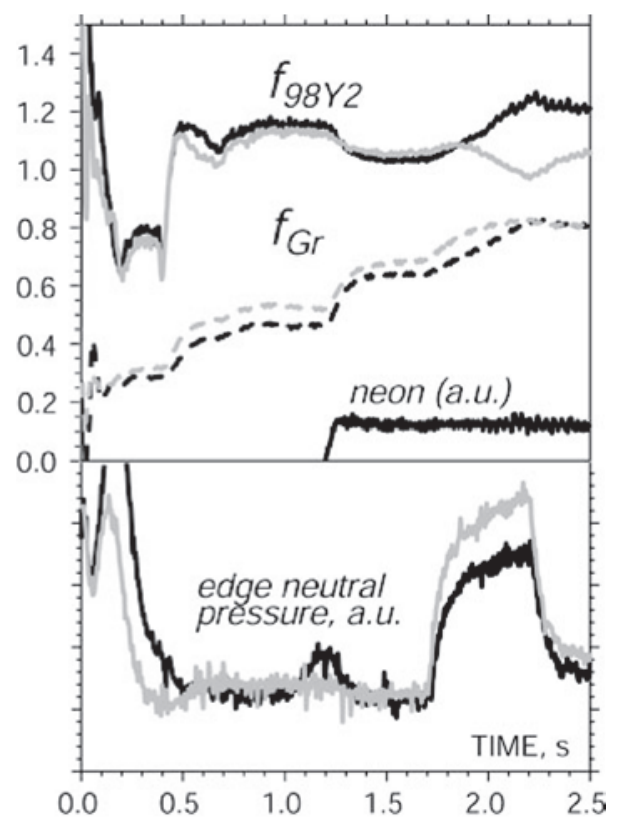

Figure 2. Time traces of enhancement factor with respect to ITER98Y2 scaling, $f_{98 Y 2}$, Greenwald number, $f_{\mathrm{Gr}}$, and edge neutral pressure for two discharges fuelled with deuterium (black curves) and hydrogen (grey curves).

Figure 2 compares time traces of two discharges heated with $1 \mathrm{MW}$ of deuterium neutral beam and fuelled with deuterium (black curves) and hydrogen (grey curves). The correlation of the confinement properties to the recycling flux during the gas blowing phase (times 1.7-2.2 s) in these plasmas is similar to that observed in experiments with different intensities of deuterium puffs. The degradation of confinement in discharges fuelled with hydrogen, as seen from the trace of the enhancement factor with respect to ITER98Y2 scaling, $f_{98 Y 2}$, happens simultaneously with increasing recycling flux being proportional to the edge neutral pressure. Figure 3 shows an example of confinement degradation triggered by intense deuterium injection. In a discharge, where the intensity of the deuterium puff was strongly increased at a time of $1.9 \mathrm{~s}$, the degradation of the plasma energy content also occurs simultaneously with the intensification of the recycling flux (see also figure 2).

The correlation of the global confinement with the recycling flux observed in both cases supports a similar mechanism leading to confinement degradation. In [5], it was demonstrated that a strong deuterium puff affects the core confinement through an intensification of edge turbulence, which is the cause for an increase in the recycling flux. Higher edge transport leads to a pump out of the puffed impurity and a reduction of its content in the plasma core. This causes a restart of ITG instability suppressed by the neon injection, the density profile becomes flatter, as is observed experimentally under conditions of strong deuterium puffs, and energy confinement degrades.

Figure 4 presents the profiles of density and effective charge for two discharges shown in figure 2 at times $1.5 \mathrm{~s}$ (before the puff) and $2.0 \mathrm{~s}$ (during the puff). For the evaluation of the electron density profiles a nine-channel interferometer was used. The electron density is measured with an accuracy of $\approx 10 \%$. The $Z_{\text {eff }}$ profiles are obtained by measuring the bremsstrahlung in the visible range, by scanning one poloidal cross-section with a tilting mirror. The main source of error on the $Z_{\text {eff }}$ profile was assumed to be the uncertainty of the 


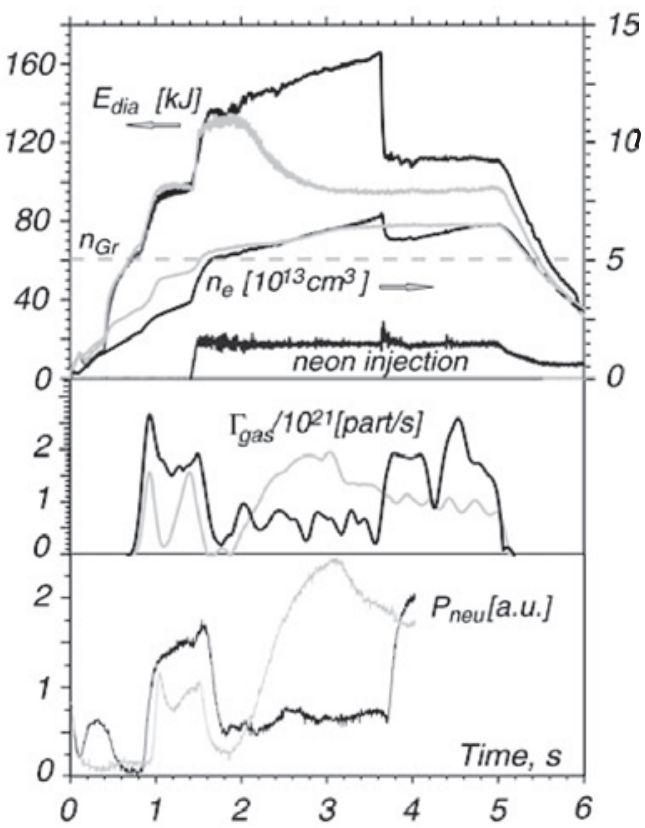

Figure 3. Influence of intensity of the deuterium puff on the RI mode performance; time traces for diamagnetic energy, $E_{\text {dia }}$, electron density, deuterium influx intensity and edge neutral pressure.
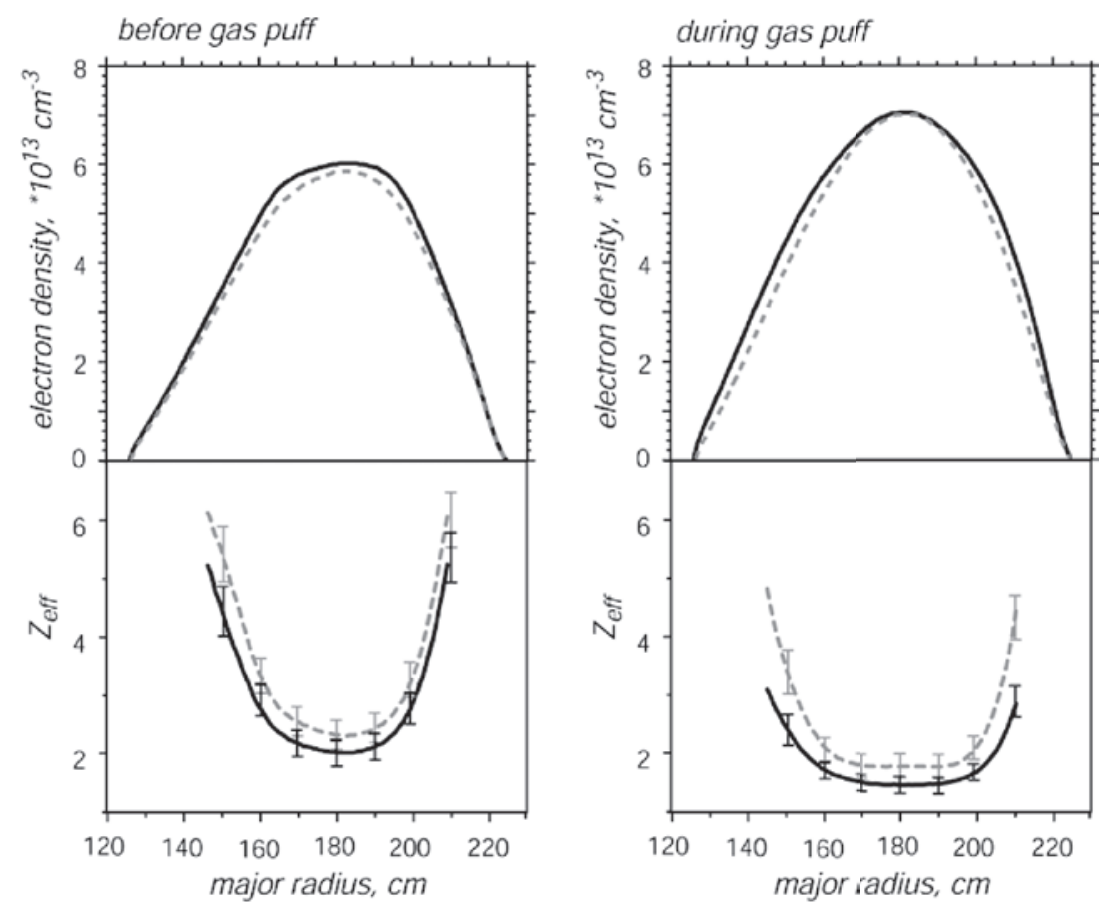

Figure 4. Profiles of density and effective charge for two discharges shown in figure 2 . The discharge fuelled with hydrogen is represented by solid curves and that with deuterium-by dashed ones 
ratio of the edge bremsstrahlung emissivity to the total continuum emissivity. To quantify the effect of this uncertainty on the profile we used the technique described in [15], a random error was introduced in the edge of an arbitrary emissivity profile. This adapted profile was then integrated along a set of chords from which a new profile was reconstructed. It was seen that, although the introduced error on the original edge emissivity profile was considerable, the effect on the resulting $Z_{\text {eff }}$ profile outside the edge region was only limited. Therefore, the conclusion on the general tendency of $Z_{\text {eff }}$ in the core plasma seems to be justified with an error of $20 \%$.

The most remarkable difference between discharges fuelled with deuterium (dashed curves) and hydrogen (solid curves) occurs with respect to the level of $Z_{\text {eff }}$, which decreases noticeably in the case of hydrogen puffing. In both discharges, the shape of the density profile does not change during the density ramp by puffing and the profiles remain strongly peaked. Although the impurity content reduces in discharge fuelled with hydrogen, the critical temperature gradients for the ITG mode do not change significantly and remain larger than the measured ones. Thus, one cannot interpret the confinement degradation as a result of the restart of ITG instability.

As was mentioned earlier, transport in the RI mode is governed mainly by the DTE instability [6] and depends on the effective collision frequency of trapped electrons, $\nu_{e, \text { eff }}=R / \tau_{\mathrm{ei}} r$ :

$$
D^{\mathrm{DTE}} \approx \rho_{s} f_{\mathrm{tr}} \eta_{e} \omega_{*} \frac{\omega_{*}}{v_{e, \mathrm{eff}}}
$$

where $\tau_{\mathrm{ei}}$ is the electron-ion collision time, $R$ and $r$ are the major and minor plasma radii, respectively, $\rho_{s}$ the ion Larmor radius, $f_{\text {tr }}$ the fraction of trapped particles, $\omega_{*}=$ $\left(c T_{e} k_{\perp, \max } / e B\right)\left(-\mathrm{d} \ln n_{e} / \mathrm{d} r\right)$ the drift frequency and $\eta_{e}$ the ratio of $e$-folding lengths for the electron density and temperature. The reduction of $Z_{\text {eff }}$ during the hydrogen puff leads to a decrease of the collision frequency and a proportional increase of the transport driven by the DTE unstable modes. This fact might explain the confinement deterioration observed experimentally.

In order to check this possibility, we have performed computations with the onedimensional transport code RITM [4]. This code was earlier successfully applied to interpret the effect of strong deuterium puff on confinement in the RI mode [3].

\section{Modelling with one-dimensional RITM-code}

The one-dimensional transport code RITM allows a self-consistent simulation of the particle and heat transport in the entire cross-section of the plasma from the axis to the last closed magnetic surface (LCMS), including the very plasma edge where particle sources, due to recycling, are of importance. It solves fluid-type equations for electrons and impurity ions, in which the diffusive and convective components of the total fluxes are included. For the background ions the quasi-neutrality conditions are assumed. The transport coefficients are determined in mixing length approximation from the growth rate and perpendicular wavenumber of the most unstable modes [3-5]. Three kinds of instabilities are normally contributions to be taken into account. In the plasma core, the largest are from DTE (see equation (1)) and ITG unstable modes: the characteristic diffusion coefficients for electrons and impurity ions are

$$
\begin{aligned}
D^{\mathrm{ITG}} \approx & \frac{c T_{e}}{k_{\perp}^{\mathrm{ITG}} e B} \cdot\left[\left(-\frac{\mathrm{d} \ln T_{i}}{\mathrm{~d} r}+\frac{2}{3} \frac{\mathrm{d} \ln n_{e}}{\mathrm{~d} r}\right)\left(Z_{\mathrm{eff}} R\right)^{-1}\right. \\
& \left.-\frac{1}{8}\left(\frac{\mathrm{d} \ln n_{e}}{\mathrm{~d} r}+\frac{2}{R}\right)^{2}+\frac{20}{9}\left(\frac{1}{R Z_{\mathrm{eff}}}\right)^{2}\right]^{1 / 2} .
\end{aligned}
$$


At the plasma edge, the transport is governed mainly by drift resistive ballooning (DRB) instability [11] with:

$$
D_{\mathrm{DRB}} \approx\left(2 \pi q \rho_{e}\right)^{2} v_{e} \frac{R}{L_{n}} .
$$

The anomalous particle pinch velocity, assumed for electrons, is mainly due to the magnetic shear $[12,13]$ :

$$
V=\left(D^{\mathrm{ITG}} \cdot f_{\mathrm{tr}} \cdot \varepsilon+D^{\mathrm{DTE}}\right) \frac{\mathrm{d} \ln q}{\mathrm{~d} r}
$$

where $q$ is a safety factor. The small factor $\varepsilon=4 r / 3 R$ reflects a relatively weak effect on convection of instabilities caused by ion dynamics, in particular, ITG instability $[12,13]$. For impurity particles we adopt a neoclassical pinch velocity, which is caused by the density and temperature gradients of the background ions [14].

The influx of puffed hydrogen molecules does not exceed the percentage of the recycling deuterium flux. Therefore, deuterium is always considered as a single background species in the plasma. However, at the local position of puffing the concentration of the hydrogen particles can be close to $100 \%$. Recently, it was demonstrated that edge anomalous transport can be significantly influenced by the local plasma state in the vicinity of the gas puff location [8]. In order to explain the higher recycling flux observed in the experiment with hydrogen fuelling (see figure 2), we have presumed that the edge transport is somewhat higher under these conditions than in the case of deuterium puff when it is described by equation (3). Figure 5 shows the time traces of density, edge diffusion, recycling flux and diamagnetic energy modelled with RITM code. The same intensity of neutral gas puffing was assumed, but in the computation represented by solid curves, simultaneously with the gas puffing starting at $0.7 \mathrm{~s}$, a $15 \%$ increase in the edge transport was assumed. In both discharges the gas puff leads to the same increase of the line-averaged density. In the case with the higher edge transport the latter causes larger recycling flux and lower impurity density. Figure 6 shows the final profiles of the electron density, $Z_{\text {eff }}$ and transport coefficients induced by different instabilities for discharges shown in figure 5. The solid curves obtained with the edge particle diffusion, which is $15 \%$ larger than that given in equation (3), demonstrate the reduction of $Z_{\text {eff }}$ and a proportional increase of DTE-induced transport.

The drop of $Z_{\text {eff }}$ can be understood if we take into account that in a steady state the influx of puffed neon neutrals should be balanced by the outflow of the ions. The latter can be estimated as [9] $D_{\perp}\left(n_{\mathrm{I}} / l_{0}\right)$, where $D_{\perp}$ is the edge particle diffusivity, $l_{0}$ the penetration depth of impurity neutrals and $n_{\mathrm{I}}$ the impurity ion density in the plasma. Thus, by increasing the edge transport one diminishes the impurity content. This leads to a growth of the transport in the plasma core due to DTE unstable modes and to the reduction of the plasma energy content.

The contribution from ITG instability to the total transport does not change noticeably because the peaked shape of the density profile is not affected by the small change in the edge transport. Therefore, ITG turbulence remains suppressed by the density gradient in the main part of the minor radius.

It is reasonable to pose the question: is there any possibility to avoid the negative impact of hydrogen fuelling on confinement? If the reduction of confinement occurs due to a decrease of $Z_{\text {eff }}$, the obvious way to prevent this is to keep impurity concentration on the same level as before the hydrogen puff.

To simulate this possibility, we have done the following numerical experiment by using the RITM code: in a discharge with a higher edge transport during the gas puff phase, which represents the case of hydrogen injection, the level of impurity puffing was doubled. Figure 7 shows the time traces of edge diffusion and recycling flux. In computations represented by the 


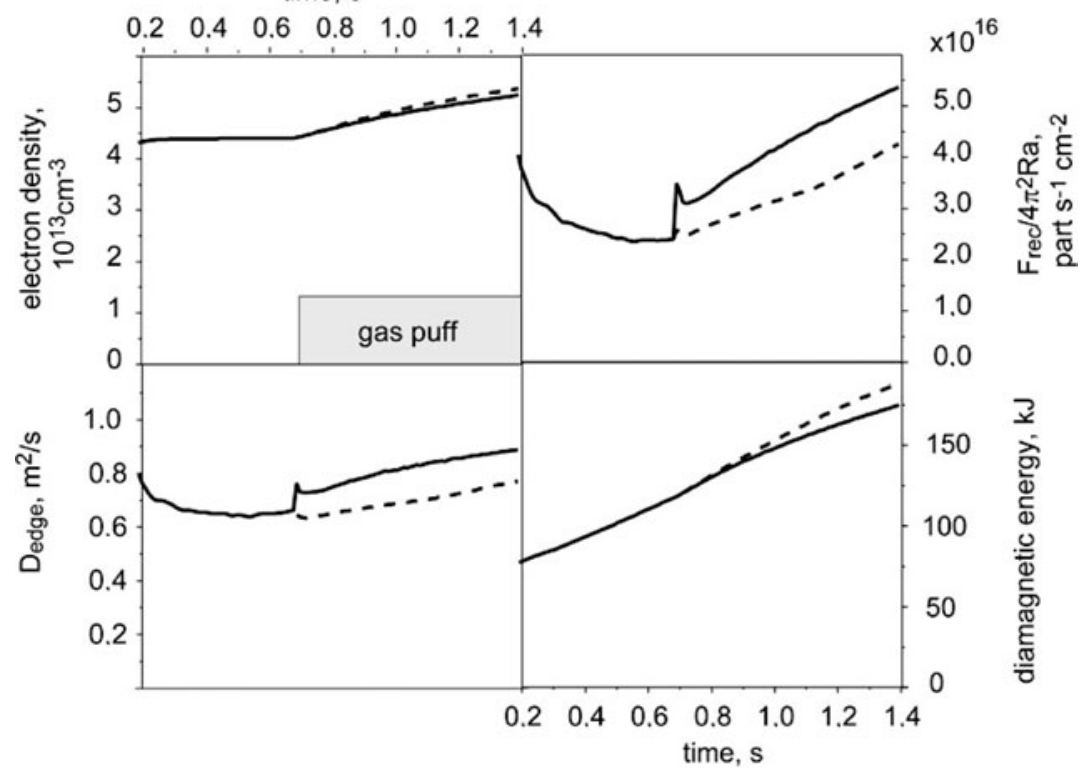

Figure 5. Time traces of density, edge diffusion, recycling flux and diamagnetic energy modelled with RITM code; during the gas blowing phase a $15 \%$ higher edge transport was assumed in computations represented by a solid curve.

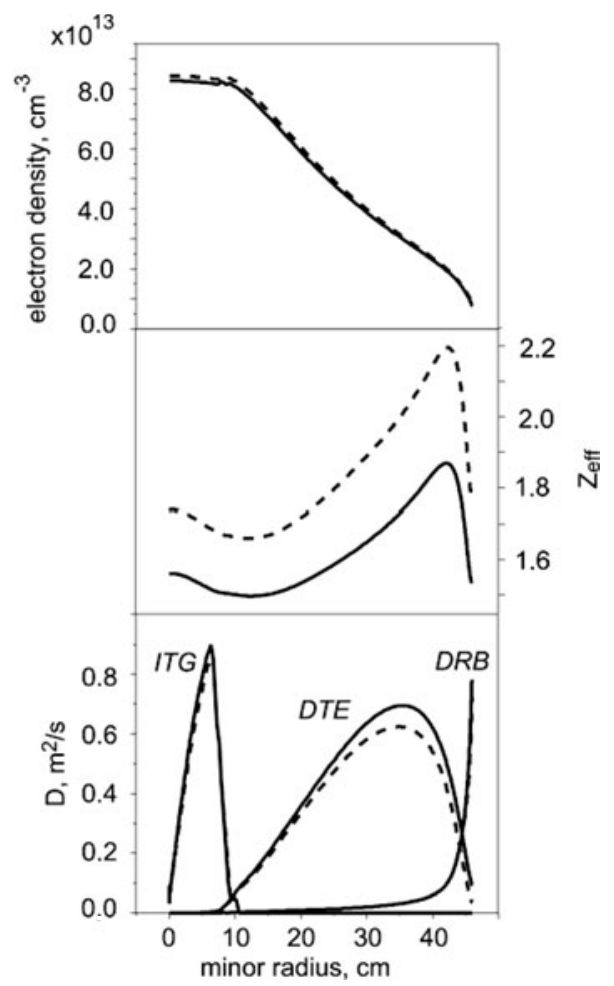

Figure 6. Final profiles of density, $Z_{\text {eff }}$ and transport coefficients from ITG, DTE and DRB instabilities for discharges shown in figure 5 ; solid curve corresponds to computation made with higher edge transport. 

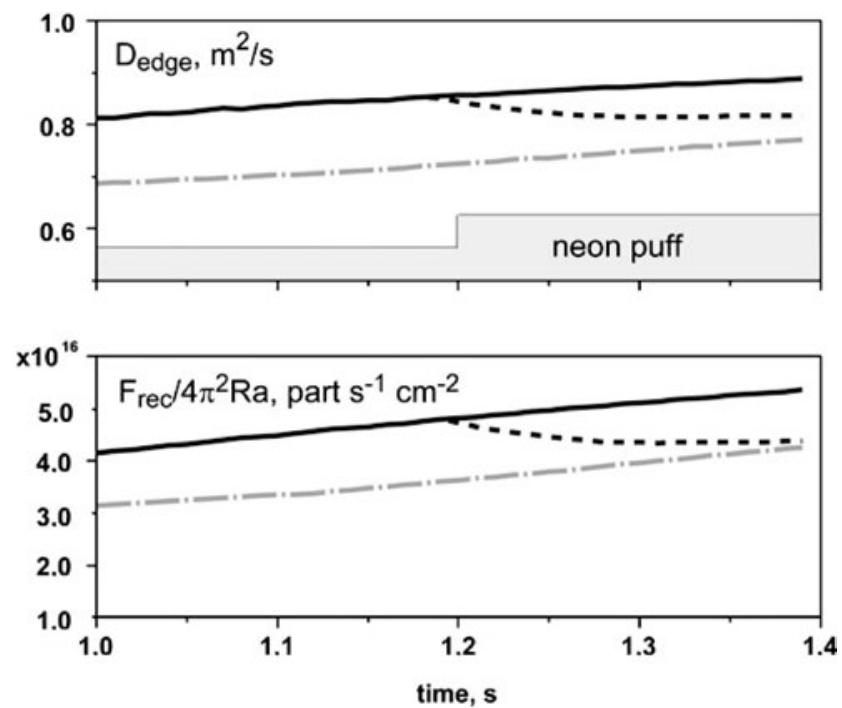

Figure 7. Time traces of edge diffusion and recycling flux during the gas blowing phase (increased edge transport was assumed in computations represented by black curves; for dashed black curve the amount of neon influx was doubled at $t=1.2 \mathrm{~s}$ ).

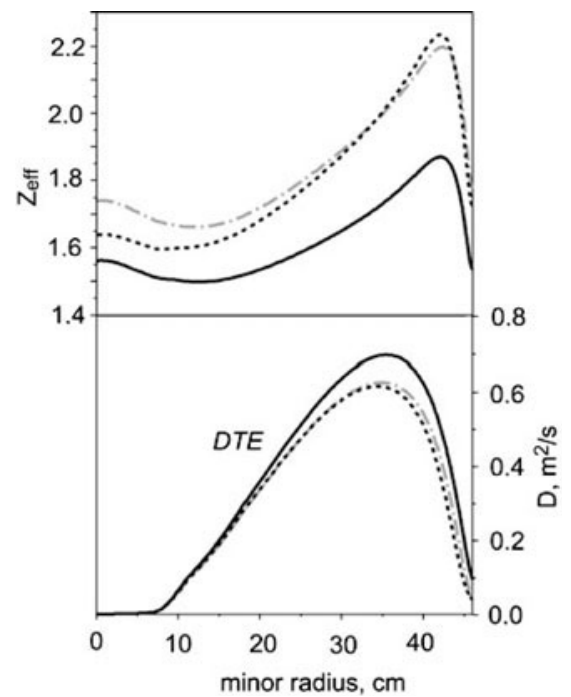

Figure 8. Final profiles of $Z_{\text {eff }}$ and the DTE-diffusion coefficients for discharges shown in figure 7.

black dashed curve at time $=1.2 \mathrm{~s}$, the neon puff was increased by a factor of two. This leads to the reduction of the edge transport and the recycling flux towards the level observed in discharge without confinement degradation. As a result of decreased diffusion and under conditions of weakly changing pinch velocity (assumed neoclassical for impurities), $Z_{\text {eff }}$ recovers to its previous level (see figure 8) and transport driven by DTE instability decreases. This procedure allows hydrogen fuelled plasmas to recover the confinement properties of discharges with deuterium puffs. 


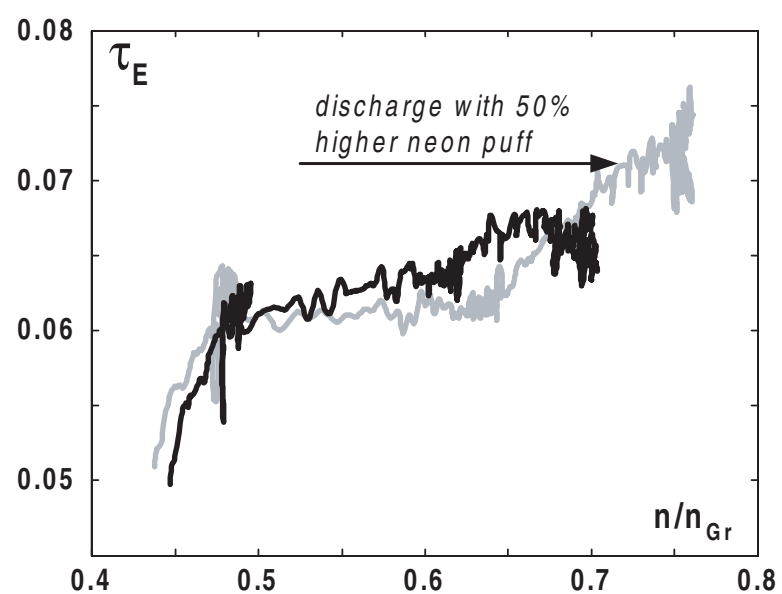

Figure 9. Energy confinement time versus Greenwald number for two discharges fuelled with hydrogen and differ by intensity of background neon puff.

To test this idea tentatively new experiments are needed. But already from previous experiments it is possible to conclude that the energy confinement in discharges with more intense neon puffing is less affected by hydrogen injection. Figure 9 compares the evolution of energy confinement time versus Greenwald number for two discharges fuelled with hydrogen and differ by intensity of background neon puff. In discharge represented by the grey curve, the neon injection rate is $50 \%$ higher than in discharge shown by the black curve. The puff of the same amount of hydrogen gas allows to obtain higher energy confinement and also higher density in the discharge with a higher neon puffing rate. It confirms the results obtained with the RITM code: by a control of the impurity concentration it is possible to avoid or significantly reduce the confinement degradation provoked by hydrogen fuelling.

\section{Summary}

The RI mode experiments with external hydrogen and deuterium puffing into deuterium plasmas were considered in this paper. The relatively small amount of hydrogen injected into the plasma does not allow interpretation of confinement reduction as a result of decreasing atomic mass of the working gas. Such a reduction occurs due to increased DTE-driven transport in the plasma core. This happens because a higher edge transport during the hydrogen puff phase leads to the reduction of impurity content in the plasma core and proportional increase of DTE-governed transport.

The control of the $Z_{\text {eff }}$ level by an intensification of the neon injection during the gas puffing phase is a possible way to avoid the reduction of confinement under hydrogen puff conditions.

\section{References}

[1] Van Wassenhove G et al 2002 29th EPS Conf. on Plasma Physics and Controlled Fusion (Montreux, Switzerland) p 2.057

[2] Unterberg B et al 2000 IAEA-CN-77/EX5/2

[3] Kalupin D et al 2001 Plasma Phys. Control. Fusion 43945

[4] Tokar M Z 1994 Plasma Phys. Control. Fusion 361819

[5] Kalupin D PhD Thesis Berichte des Forschungszentrums Jülich, No 3985

[6] Kadomtsev B B and Pogutse O P 1971 Nucl. Fusion 1167 
[7] Tokar M Z et al 2000 Phys. Rev. Lett. 84895

[8] Tokar M Z et al 2002 29th Conf. on Plasma Physics and Controlled Fusion (Montreux, 17-21 June 2002) (ECA) vol 26B, p 1-097

[9] Engelhardt W and Feneberg W 1978 J. Nucl. Mater. 76 \& 77518

[10] Dominguez R R and Waltz R E 1987 Nucl. Fusion 2765

[11] Gusdar P N et al 1993 Phys. Fluids B 53712

[12] Isichenko M B et al 1995 Phys. Rev. Lett. 744436

[13] Baker D R and Rosenbluth M N 1998 Phys. Plasmas 52936

[14] Wenzel K W and Sigmar D J 1990 Nucl. Fusion 301117

[15] Telesca G et al 1992 ICPP, Innsbruck, Austria, 29 June-3 July 1992, Part II, pp 1147-50 